\title{
Organisation, réarrangement et diversité des gènes TRG gamma des lymphocytes $T$ humains
}

\section{Marie-Paule Lefranc Professeur à l'université de Montpellier II}

\section{ADRESSE}

M.-P. Lefranc : laboratoire d'immunogénétique, U.A. Cnrs 1191 génétique moléculaire, université des sciences et techniques du Languedoc, place E. Bataillon, 34060 Montpellier es gènes codant les châ̂nes $\alpha$ et $\beta$ du récepteur des antigènes des lymphocytes T (TCR $\alpha$ et $\beta$ ) subissent, dans ces cellules, des réarrangements spécifiques analogues à ceux des gènes des immunoglobulines dans les lymphocytes B (médecine/sciences $n^{\circ} 6$, vol. 2 , p. 296 et 304). Un troisième type de gènes, désigné TRG $\gamma$ (T-cell rearranging gene gamma) a été récemment isolé chez la souris [1, 2] et chez l'homme $[3,4]$. Les gènes TRG $\gamma$ humains, localisés sur le chromosome 7 (7p15) $[4,5]$, sont, comme les gènes TCR $\alpha$ et $\beta$, réarrangés d'une manière spécifique dans les lymphocytes $T$.

Bien qu'aucune donnée immunologique n'ait laissé prévoir à ce jour l'existence des gènes TRG $\gamma$ et que la fonction de la protéine gamma codée par ces gènes soit encore tout à fait inconnue, il semblerait qu'il puisse s'agir d'un deuxième type de récepteurs $[6,7]$.

\section{Organisation des gènes TRG $\gamma$ humains}

Avant même que la protéine gamma soit identifiée $[6,7]$, on savait, par l'analyse structurale des gènes et de leur réarrangement chez la souris $[1,2]$ et chez l'homme [3, 4, 8-10], qu'elle comportait une région variable $(\mathrm{V})$ et une région constante $(\mathrm{C})$. Chez l'homme, deux gènes, $\mathrm{C} \gamma 1$ et $\mathrm{C} \gamma 2$, peuvent coder pour la région constante gamma $[3,4]$; ces gènes sont situés en tandem, à 16 kilobases l'un de l'autre [3] (figure 1). Le gène $\mathrm{C} \gamma 1$ humain comprend trois exons, comme les gènes $\mathrm{C}_{\boldsymbol{\gamma}}$ de souris [2], mais les introns sont plus importants et le gène $\mathrm{C} \gamma 1$ occupe près $\mathrm{de}$ 6 kilobases. Le gène $\mathrm{C} \gamma 2$ s'étend sur une distance encore plus grande (9,4 kilobases), et comporte quatre exons par suite d'une duplication de la région comprenant l'exon 2 [11] (duplication indiquée par un trait horizontal 
Chromosome 7

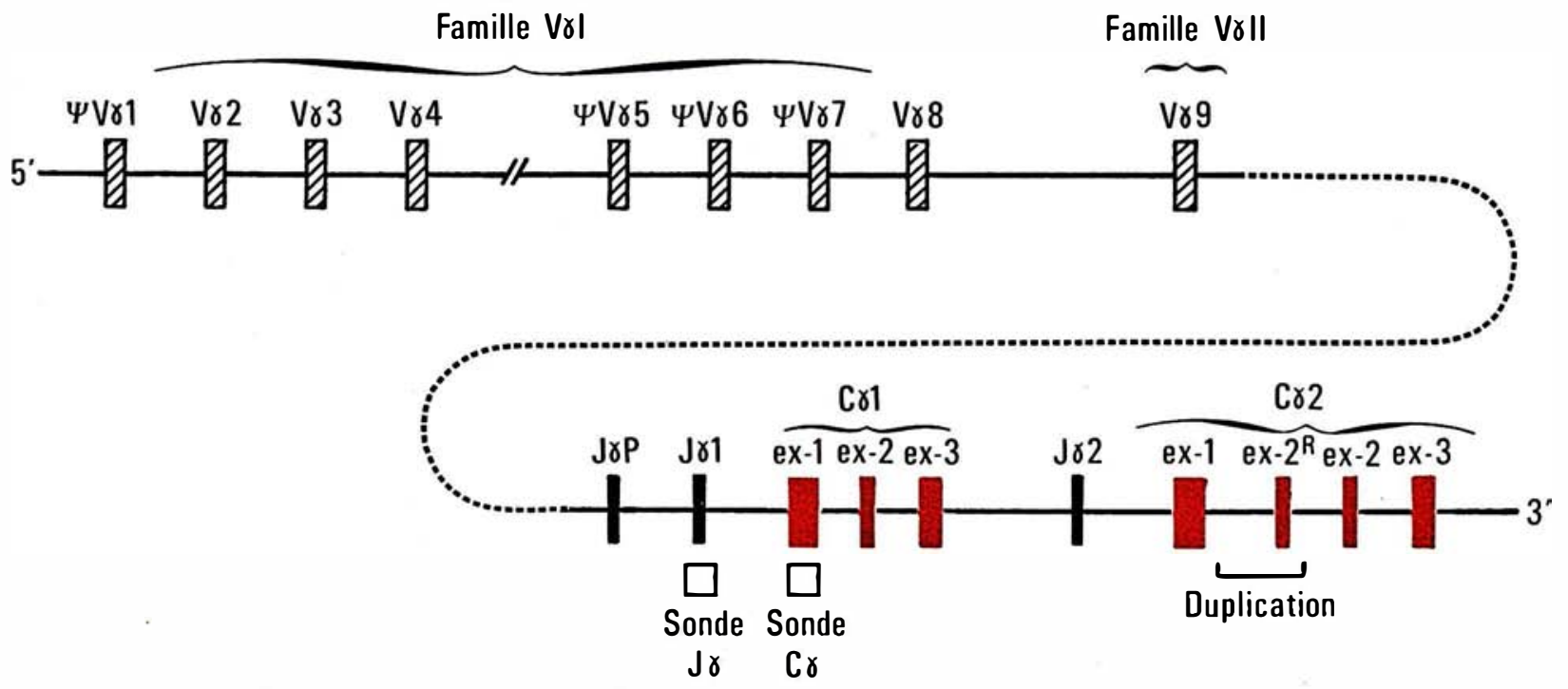

Figure 1. Organisation du locus TRG $\gamma$ humain. Deux gènes $C_{\gamma}\left(C_{\gamma} 1\right.$ et $\left.C_{\gamma}\right)$ ont été identifiés [3, 4]. Ces gènes sont en tandem à 16 kilobases l'un de l'autre [3]. Chacun d'eux est précédé d'au moins un segment Jy [8]. Un autre segment $J_{\gamma}\left(J_{\gamma} P\right)$ situé à 4 kilobases en amont de $J_{\gamma} 1$ [10] est plus rarement utilisé. Le gène $C_{\gamma 1}$ comprend trois exons (ex-1, ex-2 et ex-3). Le gène $C_{\gamma} 2$ en comporte quatre par suite d'une duplication de la région comprenant l'exon 2 [11] (cette duplication est indiquée par un trait horizontal, l'exon 2 dupliqué est noté " ex-2R "). 9 gènes $V_{\gamma}$ appartenant à deux familles différentes ont, à ce jour, été localisés sur une longueur de 54 kilobases. La famille $V_{\gamma} l$ comporte 4 gènes fonctionnels et 4 pseudogènes. La famille $V \gamma / l$ semble n'avoir qu'un seul gène [10]. II semble qu'il $y$ ait au total une douzaine de gènes $V_{\gamma}$ appartenant à 4 familles différentes [M.-P. Lefranc et al, en cours].

sur la figure 1 ; l'exon 2 dupliqué est noté "ex- $\left.2^{R} "\right)$. A 4 kilobases, en amont (en $5^{\prime}$ ) de chaque gène $\mathrm{C} \gamma$, a été identifié au moins un segment Jy ( $J$ pour "jonction ") [8] ; ce segment code pour seize acides aminés de la région variable, la majeure partie de celle-ci étant elle-même codée par un des gènes $\mathrm{V} \gamma$ (figure 1) situés plus en amont sur le chromosome 7 [10] mais à une distance encore inconnue.

\section{Jonction V-J}

Dans les cellules $T$, un gène $V \gamma$ devient contigu à un segment $\mathrm{J} \gamma$, cette jonction résultant d'un réar$m / s n^{\circ} 3$ ool. 3, mars 87 rangement des séquences d'ADN correspondantes qui s'accompagne de la délétion du fragment d'ADN initialement présent entre ces deux segments $\mathrm{V} \gamma$ et $\mathrm{J} \gamma$ (figure 2). Chacun des deux segments J $\gamma$ peut être impliqué dans un réarrangement (figure 2) [8] et les 2 gènes $\mathrm{C} \gamma$ semblent fonctionnels [11]. La jonction d'un gène $\mathrm{V} \gamma$ au segment $\mathrm{J} \gamma 2$ s'accompagne de la délétion non seulement des gènes $\mathrm{V} \gamma$ situés en aval mais aussi de la région $\mathrm{C}_{\gamma} 1$ (figure 2). Une telle délétion s'observe également dans le locus TCR $\beta$ où il existe deux gènes $C \beta$ en tandem.

Comme pour les chaînes des immunoglobulines et les chaînes
TCR $\alpha$ et $\beta$, le réarrangement entre $\mathrm{V} \gamma$ et $\mathrm{J} \gamma$ se fait grâce à la présence de signaux de jonction heptamère-nonamère (figure 3 ). Chaque gène $\mathrm{V} \gamma$ humain non réarrangé est suivi par un heptamère de séquence CACAGTG et un nonamère CTGAAAATC, séparé par un intervalle de 23 nucléotides [10]. De la même manière, les segments $\mathrm{J} \gamma$ sont précédés d'un nonamère AGTTTTTGA et d'un heptamère CACTGTG séparé par 12 nucléotides [8]. La règle dite 12/23 est respectée ; elle implique qu'une recombinaison efficace fait intervenir deux segments d'ADN, l'un avec un intervalle de 12 


\section{RÉFÉRENCES}

1. Saito H, Kranz DM, Takagaki Y, Hayday AC, Eisen HN, Tonegawa S. Complete primary structure of a heterodimeric T-cell receptor deduced from cDNA sequences. Nature $1984 ; 309: 757-62$.

2. Hayday AC, Saito H, Gillies SD, et al. Structure, organization and somatic rearrangement of T cell gamma genes. Cell 1985 ; 40 : 259-69.

3. Lefranc MP, Rabbitts TH. Two tandemly organized human genes encoding the T-cell $\gamma$ constant region sequences show multiple rearrangement in different $\mathrm{T}$-cell types. Nature 1985 ; $316: 464-6$.

4. Murre C, Waldmann RA, Morton CC, et al. Human $\gamma$-chain genes are rearranged in leukaemic $T$ cells and map to the short arm of chromosome 7 . Nature $1985 ; 316: 549-52$

5. Rabbitts TH, Lefranc MP, Stinson MA, et al. The chromosomal location of T-cell receptor genes and a $T$ cell rearranging gene : possible correlation with specific translocations in human T cell leukaemia. EMBOJ $1985 ; 4$ : 1461-5.

6. Brenner MB, McLean J, Dialynas DP, et al. Identification of a putative second T-cell receptor. Nature $1986 ; 322$ : 145-9.

7. Bank I, DePinho RA, Brenner MB, Cassimeris J, Alt FW, Chess L. A functional T3 molecule associated with a novel heterodimer on the surface of immature human thymocytes. Nature $1986 ; 322: 179-81$.

8. Lefranc MP, Forster A, Rabbitts TH. Rearrangement of two distinct $T$-cell $\gamma$-chain variable-region genes in human DNA. Nature $1986 ; 319: 420-2$

9. Quertemous T, Murre C, Dialynas D, et al. Human T-cell $\gamma$ chain genes : Organization, diversity, and rearrangement. Science 1986; 231 : 252-5.

10. Lefranc MP, Forster A, Baer R, Stinson MA, Rabbitts TH. Diversity and rearrangement of the human $T$ cell rearranging $\gamma$ genes : nine germ-line variable genes belonging to two subgroups. Cell $1986 ; 45: 237-46$.

11. Lefranc MP, Forster A, Rabbitts TH. Genetic polymorphism and exon changes of human $\mathrm{T}$ cell rearranging $\gamma$ constant region genes revealed in genomic sequences. Proc Natl Acad Sci USA 1986 ; 83 : 9596-600.

12. Early P, Huang H, Davis M, Calame K, Hood L. An immunoglobulin heavy chain variable region gene is generated from three segments of DNA : CH, D and JH. Cell $1980 ; 19: 981-92$.

13. Iwamoto A, Rupp F, Ohashi PS, et al. T cell-specific $\gamma$ genes in C57BL/10 mice. $J$ Exp Med 1986 ; 163 : 1203-12.

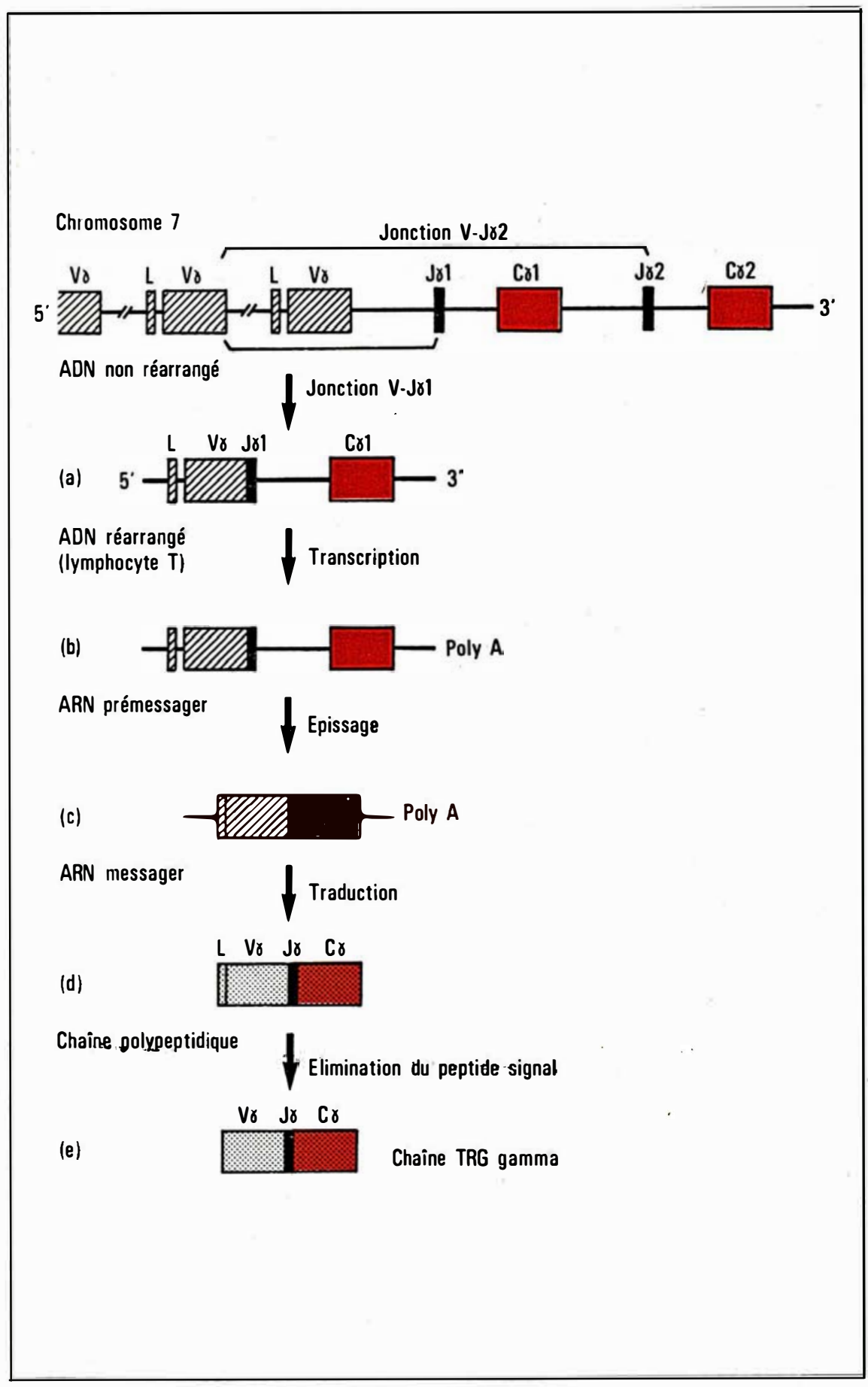

Figure 2. Réarrangement des gènes TRG $\gamma$ humains et synthèse d'une chaîno gamma. La synthèse de la chaîne TRG gamma est semblable à celle des chaînes légères et lourdes des immunoglobulines et des chaînes TCR $\alpha$ et $\beta$ du récepteur $T$. Un gène $V_{\gamma}$ avec sa séquence leader $L$ est recombiné avec un segment $J_{\gamma}$ pour former avec le gène $C_{\gamma}\left(C_{\gamma} 1\right.$ ou $\left.C_{\gamma} 2\right)$ un gène gamma actif (a). Le gène complet $V_{\gamma}-J_{\gamma}-C_{\gamma}$ est transcrit en un ARN prémessager (b), les introns sont excisés de façon à produire un ARN messager mature (c), qui est alors traduit en une chaîne polypeptidique (d). Le peptide signal $L$ est éliminé (e).

Note : les exons des gènes $C_{\gamma}$ (figure 1 ) n'ont pas été représentés pour simplifier le schéma. Les introns correspondants sont épissés lors de la maturation de I'ARN messager. 


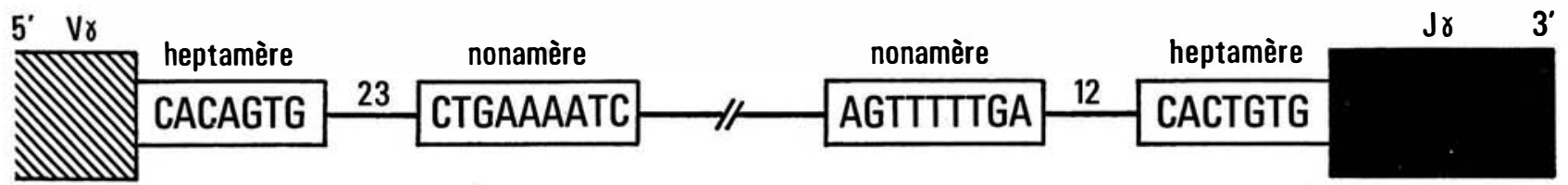

Figure 3. Séquences consensus et représentation schématique des signaux de jonction $V_{\gamma}-J_{\gamma}$ chez l'homme. Comme pour les chaînes des immunoglobulines et les chaînes TCR $\alpha$ et $\beta$, le réarrangement entre $V_{\gamma}$ et $J_{\gamma}$ se fait grâce à la présence de signaux de jonction heptamère-nonamère respectivement situés en aval (en $\left.3^{\prime}\right)$ du gène $V_{\gamma}[10]$ et en amont (en $\left.5^{\prime}\right)$ du segment $J_{\gamma}[8]$.

nucléotides et l'autre de 23 nucléotides entre les heptamères et nonamères [12].

\section{Familles de gènes $\boldsymbol{V}_{\boldsymbol{\gamma}}$}

Neuf gènes $V \gamma$ appartenant à deux familles différentes ont été à ce jour séquencés et localisés sur deux fragments d'ADN d'une longueur totale de 54 kilobases

$[8,10]$. (figure 1). Huit gènes appartiennent à une même famille $(\mathrm{V} \gamma \mathrm{I})$, quatre d'entre eux sont fonctionnels tandis que les quatre autres sont des pseudogènes pour des raisons diverses : ainsi, $\mathrm{V} \gamma 1$ comporte une délétion de 14 paires de bases entre les signaux de reconnaissance heptamère et nonamère nécessaires au réarrangement, $\mathrm{V} \gamma 5$ a un codon stop

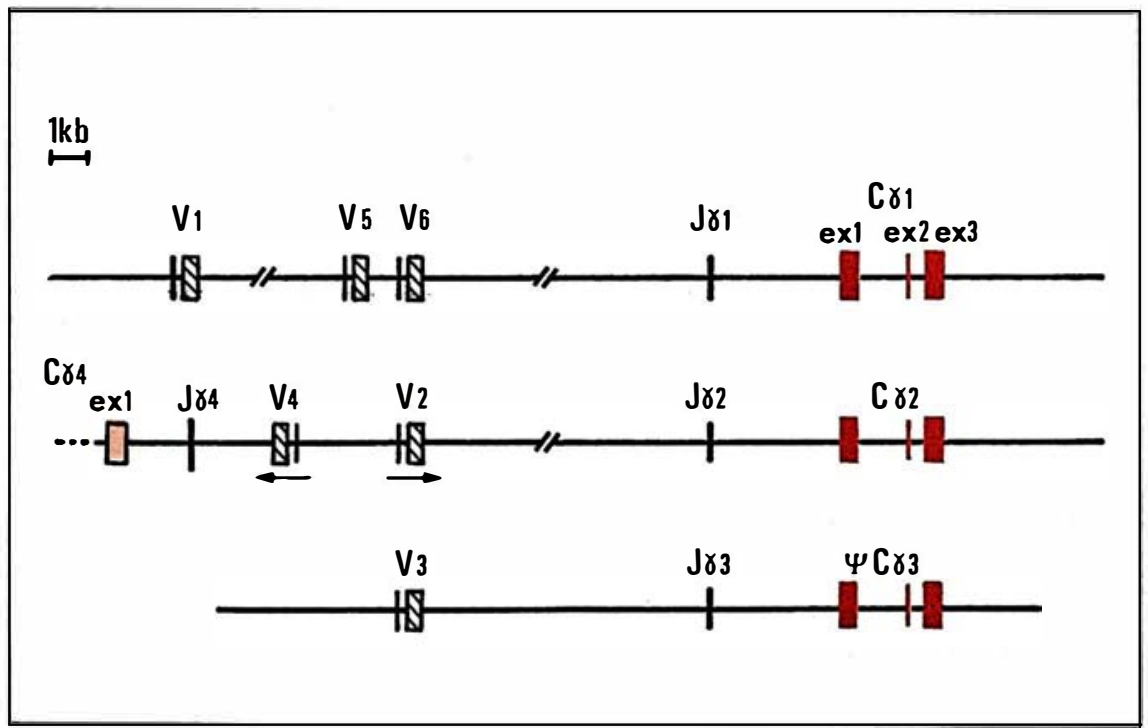

Figure 4. Organisation du locus TRG $\gamma$ chez la souris. Trois gènes $\boldsymbol{C}_{\gamma}$ et trois gènes $V_{\gamma}$ cnt $d^{\prime}$ abord été identifiés [2]. Récemment, le locus TRG $\gamma$ de la souris s'est révélé plus complexe. Un quatrième gène $C_{\gamma} 4$ et trois nouveaux $V_{\gamma}$ ont été mis en évidence [13-17]. Les gènes $V 2$ et $V 4$ se réarrangent respectivement avec $J_{\gamma 2}$ et $J_{\gamma} 4$. L'orientation de leur transcription est en sens opposé et indiquée par des flèches. La localisation et l'orientation des trois gènes $V 1, V 5$ et $V 6$ en amont de $C_{\gamma} 1$ restent à préciser. Les principaux réarrangements sont $V 1-J_{\gamma} 1$ et $V 2-J_{\gamma} 2 . C_{\gamma} 3$ est un pseudogène et n'est pas fonctionnel [2]. Par ailleurs, V3, $J_{\gamma} 3$ et $C_{\gamma} 3$ sont absents du génome de certaines souches de souris [13, 15].

$m / s n^{\circ} 3$ vol. 3 , mars 87 peu après le codon d'initiation, $\gamma 6$ et $\mathrm{V} \gamma 7$ ont une délétion de un nucléotide entraînant un mauvais cadre de lecture [10]. La seconde famille, $\mathrm{V} \gamma \mathrm{II}$, semble ne comporter qu'un seul gène $(\mathrm{V} \gamma 9)$. Le pourcentage d'homologie entre les deux familles est très faible ( $30 \%$ au niveau protéique), ce qui explique qu'il n'y ait pas d'hybridation croisée entre elles [8]. D'autres gènes $V_{\gamma}$ sont en cours d'isolement, il semble qu'il y ait au total une douzaine de gènes $\mathrm{V} \gamma$ appartenant à quatre familles différentes (M.-P. Lefranc et al, en préparation). L'étude de nouveaux réarrangements devrait permettre de préciser et compléter l'organisation du locus gamma chez l'homme. Les résultats acquis à ce jour montrent que cette organisation des TRG $\gamma$ humains est différente de celle trouvée chez la souris $[1,2$, 13-17] (figure 4) mais ceci ne doit pas surprendre : des différences au moins aussi importantes existent par exemple entre le locus lambda des chaînes légères des immunoglobulines de la souris et de l'homme.

\section{Diversité des régions variables}

Lors de la maturation du lymphocyte $\mathrm{B}$, la diversité des régions variables des chaînes légères (VL) et lourdes (VH) des immunoglobulines est augmentée grâce à l'apparition de mutations somatiques ponctuelles qui affectent 
préférentiellement les gènes réarrangés VL et VH. Afin de déterminer si un tel mécanisme intervenait au niveau des chaînes TRG $\gamma$ des lymphocytes $T$, les séquences des mêmes gènes $V_{\gamma}$ non réarrangés et réarrangés ont été comparées. Aucune différence n'a été décelée (excepté dans la région dite région $\mathrm{N}$ qui sera discutée plus loin) [10]. Ainsi, contrairement aux immunoglobulines, les régions variables des chaînes TRG $\gamma$ ne présentent pas de mutations somatiques ponctuelles. Des résultats semblables ont été observés pour les chaînes TCR $\beta$.

La diversité des régions variables des chaînes gamma va donc dépendre d'autres mécanismes, d'une part du " choix " de l'un ou l'autre des gènes $\mathrm{V} \gamma$ lors du réarrangement, d'autre part de la diversification au niveau de la région $\mathrm{N}$. En effet, deux segments $\mathrm{V} \gamma$ identiques réarrangés à un même segment $\mathrm{J}$ vont présenter des différences importantes au niveau de la jonction V-J par suite de la délétion et/ou de l'addition de nucléotides au niveau de cette jonction. La terminal transférase* semble être l'enzyme responsable de cette addition de nucléotides supplémentaires [18]. Il en résulte une région, dite région $\mathrm{N}(\mathrm{N}$ pour nucléotides), qui présente une grande diversité d'un gène réarrangé à l'autre $[10,19]$. Un tel mécanisme de diversité n'est pas sans rappeler ce qui est observé au niveau de la jonction V-D-J des chaînes lourdes des immunoglobulines [18], avec une différence, cependant, puisque dans le cas des gènes TRG $\gamma$, il ne semble pas exister de segments D [10].

Un nouveau segment $J(J \gamma \mathrm{P})$ a récemment été mis en évidence à 4 kilobases en amont de $\mathrm{J} \gamma 1$ [10]. La séquence protéique de ce segment est différente de celle des segments $\mathrm{J} \gamma 1$ et $\mathrm{J} \gamma 2$ et représente donc une source de diversité supplémentaire si ce segment $\mathrm{J} \gamma \mathrm{P}$ est utilisé au cours de réarrangements fonctionnels.

Récemment, il a été démontré que des gènes réarrangés $\mathrm{VH}-\mathrm{JH}$ de chaînes lourdes d'immunoglobulines pouvaient subir un deuxième réarrangement, le gène $\mathrm{VH}$ déjà joint à un segment $\mathrm{JH}$ pouvant être remplacé par un nouveau gène $\mathrm{VH}$ situé plus en amont** $[20,21]$. Ce second réarrangement ferait intervenir une séquence TACTGTG qualifiée d' " heptamère interne " (par suite de son homologie avec l'heptamère des signaux de jonction et de sa localisation à l'intérieur même du gène $\mathrm{VH})[20,21]$. Il est intéressant de noter que cet heptamère interne est également trouvé dans les gènes $\mathrm{V} \gamma$ publiés à ce jour [8, 10], et que la possibilité d'un second réarrangement des gènes $\mathrm{V} \gamma$ existe [20].

\section{Exclusion allélique}

Les réarrangements $\mathrm{V} \gamma$-J $\gamma$ peuvent être productifs (le cadre de lecture est respecté au niveau de la jonction $V-J$ ) ou non productifs (le cadre de lecture est incorrect et aboutit à un codon stop). La présence d'un réarrangement productif sur un chromosome et d'un autre, non productif, sur le second, semble indiquer qu'une seule chaîne fonctionnelle est synthétisée, c'est-à-dire qu'il y a exclusion allélique pour les châ̂nes TRG $\gamma[10]$, comme cela est observé pour les chaînes des immunoglobulines et les chaînes TCR $\alpha$ et $\beta$.

Il est à noter qu'un réarrangement $\mathrm{V} \gamma-\mathrm{J} \gamma$ non productif n'empêche pas la transcription du gène en un ARN messager [22] ; c'est donc au stade de la traduction qu'interviendrait, dans ce cas, le mécanisme de l'exclusion allélique. D'autre part, certaines cellules $T$ semblent ne pas avoir de réarrangement productif $[10,19]$. Plusieurs hypothèses, dont celle d'un réarrangement productif devenu non fonctionnel ou celle de l'existence d'un gène $\mathrm{C} \gamma$ non encore identifié, peuvent expliquer cette situation.

\section{Réarrangements dans le thymus}

24. Foa R, Casorati G, Giubellino MC, et al. Immunoglobulin $\mathrm{T}$ cell receptor $\beta$ and TRG $\gamma$ rearrangements are associated with tenninal deoxynucleotidyl transferase expression in acute
Des préparations d'ADN de thymus de fotus âgés de 18 et 20 semaines ont montré que les réar- 
rangements précédemment décrits dans les lymphocytes $T$ périphériques sont déjà réalisés à ce niveau et à ce stade du développement fotal [10]. La présence simultanée de gènes non réarrangés et réarrangés dans le thymus indique que la population des thymocytes est en cours de différenciation. Seuls les gènes $\mathrm{V} \gamma$ fonctionnels semblent subir un réarrangement, ce qui laisse supposer l'existence, au niveau du thymus, d'un mécanisme soit de sélection des seuls gènes $\mathrm{V} \gamma$ fonctionnels, soit d'inhibition des réarrangements des pseudogènes [10]. Chacun des gènes réarrangés peut être identifié [10] par la taille des fragments de restriction obtenus par hybridation de l'ADN des thymocytes avec la sonde $\mathrm{J} \gamma$ (figure 1$)[3,8]$, après que cet ADN ait été digéré par les enzymes de restriction Bam HI, Hind III et Eco RI, puis transféré sur filtre de nitrocellulose. Les réarrangements décrits au niveau du thymus ont été retrouvés dans les lignées de cellules $T[10]$. D'autres réarrangements plus rares, observés dans certaines leucémies $\mathrm{T}$ et des lymphomes $\mathrm{T}$, sont en cours d'exploration.

\section{Clonalité}

\section{des leucémies $T$}

Les deux gènes TRG $\gamma$ montrent des réarrangements variés dans tous les lymphocytes $\mathrm{T}$ (helpers, cytotoxiques ou suppresseurs) ainsi que dans toutes les formes de leucémies T (leucémies lymphocytiques chroniques [CLL] et aiguës [ALL], leucémies prolymphocytiques [PLL], leucémies $T$ de l'adulte, syndrome de Sézary). Il est souvent difficile de démontrer la clonalité des leucémies $T$, et dans cette perspective, la sonde TCR $\beta$ s'est révélée très utile. Les gènes TRG $\gamma$ étant réarrangés de

\footnotetext{
- Teminal transférase : enzyme ajoutant des nucléotides à l'extrémité 3 ' des brins d'ADN. Cette addition ne nécessite pas de "mastrice "nucleotidique et se fait au hasard.

* Voir noudelle $m / s n^{\circ} 1$, vol. 3, p. 50.

$\mathrm{m} / \mathrm{s} n^{\circ} 3$ vol. 3, mars 87
}

manière spécifique dans tous les types de cellules $\mathrm{T}$ étudiés, la sonde $\mathrm{J} \gamma$ est également un bon indicateur de prolifération clonale des lymphocytes $\mathrm{T}$ et un outil précieux pour déterminer l'origine $T$ des leucémies et lymphomes. Il faut souligner cependant qu'un réarrangement des gènes TRG $\gamma$ est observé, d'une part, fréquemment dans des leucémies lymphoblastiques aiguës de la lignée $B$ (dont les cellules sont à un stade pré-B), ces gènes réarrangés n'étant pas ou très peu transcrits [23], d'autre part, dans quelques cas de leucémies myéloïdes aiguës (AML) positives pour la terminal transférase [24] ; toutes ces cellules présentent par ailleurs un réarrangement des chaînes lourdes d'immunoglobulines et une partie d'entre-elles un réarrangement TCR $\beta[23,24]$. On ignore à l'heure actuelle si la présence simultanée de réarrangements spécifiques des lignées $B$ et $T$ dans une même cellule a une signification physiologique ou est liée à la malignité des cellules.

\section{Expression des gènes TRG $\gamma$ et chaîne $\gamma$}

Chez la souris, il a été possible de montrer que les gènes TRG $\gamma$ sont réarrangés et transcrits très tôt au cours de l'ontogenèse thymique avant même les gènes TCR $\beta[26,27]$. A partir de ces observations, a été émise l'hypothèse d'un rôle du produit des gènes TRG $\gamma$ dans l'apprentissage de la reconnaissance des produits de classe I ou II du complexe majeur d'histocompatibilité, par les cellules $\mathrm{T}$, dans le thymus.

Chez l'homme, le taux de transcription des gènes réarrangés TRG $\gamma$ en ARN messager est très variable dans les différentes lignées établies de cellules $T$ (T.H. Rabbitts, résultats non publiés). Ce taux est également très variable dans les cellules $\mathrm{T}$ de patients atteints de leucémies lymphoblastiques aiguës (T-ALL) et aucune corrélation n'a pu être faite ni avec le stade de différenciation des cellules malignes ou l'expression des marqueurs de surface CD4 (T4) ou CD8 (T8), ni avec l'expression ou la taille des transcrits des gènes TCR $\beta$ [25].

Récemment, la chaîne gamma a été mise en évidence à la surface de certains lymphocytes $T$, dépourvus du récepteur TCR $\alpha \beta$ mais exprimant néanmoins CD3 (T3) $[6,7]$. Il a été montré que ces lymphocytes possèdent, associée à CD3, une structure clonotypique constituée de deux châ̂nes polypeptidiques différentes $[6$, 7] : l'une d'elles est la chaîne TRG $\gamma$, l'autre appelée $\delta$, n'a pas encore été caractérisée [6]. Il n'est pas exclu cependant qu'il puisse s'agir de deux chaînes $\gamma$ glycosylées de manière différente. Par ailleurs, le complexe T3 est fonctionnel puisque des anticorps anti-T3 induisent la cytotoxicité et la sécrétion d'interleukine 2 de ces lymphocytes [7], l'induction de ces fonctions immunologiques étant analogue à celle observée avec les lymphocytes $\mathrm{T}$ exprimant le récepteur TCR $\alpha \beta$. Ces résultats ainsi que ceux concernant l'organisation et les réarrangements des gènes TRG $\gamma$ sont en faveur d'un rôle de récepteur à la surface de ces lymphocytes $\mathrm{T}[6,7,28]$.

L'association entre les deux chaînes $\gamma$ et $\delta$ du dimère n'implique pas de pont disulfure [6]. Cette observation est en accord avec la séquence du gène $\mathrm{C} \gamma 2$ humain où les deux exons 2 et $2 \mathrm{R}$ (figure 1) sont dépourvus de cystéine [11]. (La cystéine présente dans l'exon 2 du gène $C \gamma 1$ de souris pourrait permettre un pont disulfure inter chaîne [1] ; cette cystéine est conservée dans l'exon 2 du gène $\mathrm{C} \gamma 1$ humain [11].)

Enfin, une nouvelle structure clonotypique (différente du dimère TCR $\alpha \beta$ et du dimère TRG $\gamma$ $\delta[6]$, mais comme eux associée au CD3) a récemment été mise en évidence à la surface de lymphocytes $T$ possédant une activité NK (" natural killer ") et isolés du sang fotal humain [29]. La caractérisation des chaînes de ce nouveau dimère devrait permettre de préciser s'il s'agit de chaînes polypeptidiques non encore décrites ou de chaînes connues mais 


\section{RÉFÉRENCES}

25. Chen Z, Le Paslier D, Loiseau $P$, Degos L, Cohen D, Sigaux F. Human T gamma gene rearrangement and expression : I. Frequent biallelic involvement of the $\mathrm{J}^{2}$ segment in T-cell acute lymphoblastic leukemia. (Soumis.)

26. Raulet DH, Garman RD, Saito H, Tonegawa S. Developmental regulation of T-cell receptor gene expression. Nature 1985 ; 314 : 103-7.

27. Snodgrass HR, Dembić $Z$, Steinmetz $M$, von Boehmer $\mathrm{H}$. Expression of T-cell antigen receptor genes during fetal development in the thymus. Nature $1985 ; 315: 232-3$.

28. Robertson M. T-cell' receptor : gamma gene product surfaces. Nature 1986 ; 322 : 110-1.

29. Moingeon $P$, Ythier A, Goubin G, et al. A unique T-cell receptor complex expressed on human fetal lymphocytes displaying naturalkiller-like activity. Nature 1986 ; 323 : 638-40.

30. Weiss A, Newton M, Crommie D. Expression of T3 in association with a molecule distinct from the T-cell antigen receptor heterodimer. Proc Natl Acad Sci USA 1986; 83 : 6998-7002.

31. Aurias A, Dutrillaux B, Buriot D, Lejeune $\mathrm{J}$. High frequency of inversion and translocations of chromosome 7 and 14 in ataxia telangiectasia. Mutat Res $1980 ; 69$ : 369-74. associées entre elles d'une manière autre que dans les dimères TCR $\alpha \beta$ et TRG $\gamma-\delta$.

\section{Conclusion}

La fonction de la chaîne polypeptidique gamma est encore inconnue. Cependant, la mise en évidence récente de cette chaîne $\gamma$ (ainsi que d'éventuelles chaînes non encore caractérisées) à la surface de lymphocytes $T$ devrait permettre, par une meilleure connaissance de ces polypeptides associés à T3 $[6,7,29,30]$ et des cellules qui les expriment, de mieux comprendre la fonction des différents lymphocytes $\mathrm{T}$ et leur ontogenèse.

Par ailleurs, des analyses cytogénétiques ont montré que les lymphocytes de patients atteints d'ataxie-télangiectasie présentaient des réarrangements chromosomiques localisés à proximité des loci TCR $\alpha, \beta$ et TRG $\gamma$. En particulier, l'inversion du chromosome 7, fréquemment observée [31], pourrait impliquer les gènes TCR $\beta$ et TRG $\gamma$ et même peut-être une recombinaison illégitime entre ces gènes (médecine/sciences, $n^{\circ} 6$, vol. 2, p. 298)

\section{Summary}

The human TRG $\gamma$ gene (Tcell rearranging gene gamma) has been shown to rearrange specifically in human $T$ cells, including helper and cytotoxic/suppressor type, as well as in all forms of T-cell leukemias. So far, two human constant region genes $(\mathrm{C} \gamma)$ have been identified, each one has at least one $\mathrm{J} \gamma$ segment. Variable (V) region genes rearrange independently with each of the $\mathrm{C} \gamma$ genes, considerable variation in human $\mathrm{V} \gamma$ genes being generated by $\mathrm{N}$ region diversity resulting from $\mathrm{VJ}$ joining. The TRG $\gamma$ gene is an indicator of clonal proliferation characteristic of T-cell leukemia, and therefore provides a useful tool for the establishment of the T-cell origin of leukemias and lymphomas.

\section{TIRÉS A PART}

M.-P. Lefranc : laboratoire d'immunogénétique, université des sciences et techniques du Languedoc, place E. Bataillon, 34060 Montpellier Cedex. 